\title{
Mortalidad en pacientes con falla intestinal en el Hospital Central del Estado, Chihuahua, México
}

\author{
Mortality in patients with intestinal failure in the \\ Central State Hospital, Chihuahua, Mexico \\ Luis Bernardo Enríquez-Sánchez, ${ }^{*}$ Marco Javier Carrillo-Gorena, ${ }^{\ddagger}$ \\ Karla Fernández-Villalobos, ${ }^{\S}$ Laura Andrea Granados-Aldaz, \\ Luis Fernando Balderrama-Miramontes, " Luis Guillermo Gallegos-Portillo," \\ Cristina Nallely Reza-Leal," Abisag González-Villa\|
}

\section{Palabras clave: \\ Falla intestinal, complicación postquirúrgica, mortalidad, cirugía abdominal. \\ Keywords: Intestinal failure, post-operative complications, mortality, abdominal surgery.}

* Médico Especialista en Cirugía General, Jefe del Departamento de Cirugía General.

${ }^{*}$ Médico Especialista en Cirugía General, Jefe de la División Quirúrgica.

${ }^{\S}$ Licenciada en Nutrición, Encargada del Departamento de Nutrición Clínica de la Unidad de Falla Intestinal. " Médico Cirujano y Partero. Departamento de Investigación en Universidad Autónoma

\section{RESUMEN}

La insuficiencia intestinal se define como "una reducción de la masa intestinal funcional por debajo de la cantidad mínima necesaria para una adecuada digestión y absorción de los alimentos”. Es una afección rara, generalmente secundaria a una cirugía abdominal mayor. Se caracteriza por una función intestinal inadecuada para la absorción de nutrientes y electrolitos que afectaría el estado nutricional y la expectativa de supervivencia sin la administración intravenosa de nutrientes y electrolitos. En las últimas décadas se ha tomado en cuenta la importancia de esta entidad con publicaciones que tratan sobre su tratamiento, mortalidad, expectativa de vida y factores relacionados; sin embargo, los datos siguen siendo insuficientes, aún más en nuestro medio. Objetivo: Describir las tasas y causas de mortalidad entre los pacientes con falla intestinal de la Unidad de Falla Intestinal del Hospital Central del Estado en Chihuahua, Chih. Material y métodos: Se realizó un estudio transversal retrospectivo en el Hospital Central del Estado con un total de 53 pacientes en un periodo de marzo de 2016 a marzo de 2018, tomando criterios de acuerdo con el tipo de falla intestinal. Resultados: Se encontró que $37 \%$ de los pacientes incluidos en el estudio (20/53) fallecieron a causa de falla intestinal. De acuerdo con su tipo de falla intestinal, la clasificación funcional tipo II fue la que obtuvo el mayor porcentaje de fallecimientos y de acuerdo con su fisiopatología se observó mayor mortalidad a causa del síndrome de intestino corto, siguiendo la dismotilidad intestinal. Conclusiones: Es importante conocer la asociación entre los principales tipos de falla intestinal y sus principales causas de mortalidad en nuestro entorno para así lograr administrar tratamientos oportunos y adecuados y con ello disminuir la mortalidad.
Intestinal insufficiency is defined as "a reduction of the functional intestinal mass below the minimum amount needed for proper digestion and absorption of food". A rare condition, usually secondary to major abdominal surgery. It is characterized by an inadequate intestinal function for the absorption of nutrients and electrolytes, which would affect the nutritional status and the expectation of survival without the intravenous administration of nutrients and electrolytes. In recent decades the importance of this entity has been taken into account, with publications that deal with its treatment, mortality, life expectancy and related factors, however, the data are still insufficient, even more so in our environment. Objective: To describe the rates and causes of mortality among patients with intestinal failure of the Intestinal Failure Unit of the Central Hospital of the State in Chihuahua, Chih. Material and methods: Patients admitted to our Intestinal Failure Unit between March 2016 and March 2018 were enrolled. We conducted a two-year retrospective cross-sectional study. Results: It was found that $37 \%$ of the patients included in the study (20/53) died due to intestinal failure. According to the intestinal failure functional classification type, type II intestinal failure was the one that carried the highest mortality rate. According to its pathophysiology classification type a greater mortality was found due to short bowel syndrome, following intestinal dysmotility. Conclusions: It is important to know the association between the main types of intestinal failure and their main causes of mortality in our environment, in order to promptly manage our patients with adequate treatments and thereby reduce mortality rate at our unit.

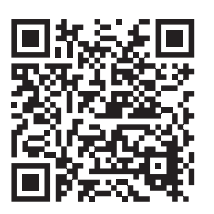

Citar como: Enríquez-Sánchez LB, Carrillo-Gorena MJ, Fernández-Villalobos K, Granados-Aldaz LA, Balderrama-Miramontes LF, Gallegos-Portillo LG, et al. Mortalidad en pacientes con falla intestinal en el Hospital Central del Estado, Chihuahua, México. Cir Gen. 2020; 42 (3): 197-202. https://dx.doi.org/10.35366/99959 
de Chihuahua, Facultad de Medicina y Ciencias Biomédicas, Chihuahua, Chih., México.

॥ Médico Pasante en Servicio Social. Departamento de Investigación en Universidad Autónoma de Chihuahua, Facultad de Medicina y Ciencias Biomédicas, Chihuahua, Chih., México.

Hospital Central del Estado, Chihuahua, Chih., México.

Recibido: 27/02/2019 Aceptado: 30/10/2019

\section{INTRODUCCIÓN}

L a falla intestinal (FI) se caracteriza por $ـ$ una reducción en la cantidad de masa de intestino funcional por debajo de lo necesario para mantener una adecuada digestión y absorción de nutrientes para lograr un estado nutricio normal.1,2 El término fue descrito por primera vez en 1981 por Fleming y Remington. De manera general, la falla intestinal puede dividirse en aguda y autolimitada, o crónica y progresiva. ${ }^{3}$

Existen varias clasificaciones y definiciones respecto a la falla intestinal, dentro de las clasificaciones más aceptadas encontramos una "clasificación funcional de la FI", la cual a su vez se divide en los tipos I, II y III; una "clasificación fisiopatológica de la FI", la cual, como su nombre lo indica, la clasifica según su mecanismo desencadenante en síndrome de intestino corto, fístula intestinal, dismotilidad intestinal, obstrucción mecánica y enfermedad malabsortiva intestinal; y una clasificación clínica de la FI crónica, la cual la divide en enfermedad no cancerosa o cáncer activo. ${ }^{4-6}$

En nuestro medio se ha observado que la causa más común de falla intestinal es la fístula entérica, lo cual corresponde con lo descrito por otros autores. ${ }^{7}$

A pesar de la existencia de estas clasificaciones en la literatura, aún no se logra llegar a un común acuerdo dentro de la comunidad científica de profesionales de la salud en cuanto a su definición y uso de las clasificaciones, lo que la convierte en un tema relevante entre los clínicos para bien de los pacientes. Sin importar qué clasificación se utilice, podemos afirmar que todas las patologías que condicionan una falla intestinal implican un importante desbalance proteico, energético e hidroelectrolítico, que en ocasiones, ya sea por sí mismo o en conjunto con otras comorbilidades, terminan en un desenlace fatal. ${ }^{8}$

\section{EPIDEMIOLOGÍA}

En un estudio retrospectivo realizado en Estados Unidos se analizaron 89 pacientes con falla intestinal, se detectó una mortalidad de 5\% (3/89) tras cirugía reconstructiva y una tasa general de mortalidad preoperatoria y postoperatoria incluyendo fallecimientos por enfermedad subyacente de $16 \%$ $(14 / 89) .^{9}$

En otro grupo de 68 pacientes con falla intestinal estudiados en Italia se observó que 22 de ellos murieron por causas secundarias a la cronicidad de la falla, de los cuales: tres murieron por causas relacionadas con la nutrición parenteral total (NPT) como sepsis asociada al uso de catéter, fallo en el acceso venoso y falla hepática, tres por no cumplir con la NPT de manera indicada, cinco por presentar alteraciones metabólicas y 11 más por causas no directamente relacionadas a la falla intestinal. ${ }^{10}$

Por desgracia en nuestro medio no hay estadísticas sobre falla intestinal en el adulto ni sobre la mortalidad asociada. Existen datos aislados acerca de las patologías que condicionan a una falla intestinal, pero debido a la falta de claridad en la definición y clasificación de ésta, resulta complicado encontrar fuentes que engloben las actuales clasificaciones.

\section{DIAGNÓSTICO Y TRATAMIENTO DE FALLA INTESTINAL}

Como se ha mencionado, aún no existe un consenso en cuanto a una clasificación mundialmente aceptada para el diagnóstico de falla intestinal, dentro de las más aceptadas existen (Tabla 1):

\section{Clasificación fisiopatológica:}

Esta clasificación está basada en el mecanismo principal, el cual, ya sea solo o en asociación con algún otro, puede determinar si un paciente desarrollará o no falla intestinal. Consiste en cinco afecciones fisiopatológicas principales que pueden originarse a partir de diversas enfermedades gastrointestinales o sistémicas: ${ }^{5}$

- Síndrome de intestino corto

- Fistula intestinal

- Dismotilidad intestinal

- Obstrucción mecánica

- Enfermedad malabsortiva intestinal 


\section{Clasificación funcional: ${ }^{4}$}

Tipo I: aguda, por lo regular autolimitada.

Tipo II: aguda prolongada, pacientes metabólicamente inestables que requieren suplementación intravenosa de semanas a meses.

Tipo III: crónica, metabólicamente inestable, requiere suplementación intravenosa de meses a años, reversible o irreversible.

\section{Clasificación clínica: ${ }^{6}$}

Esta es la clasificación más reciente, el panel de expertos de la Sociedad Europea de Nutrición Clínica y Metabolismo (ESPEN, por sus siglas en inglés: European Society for Clinical Nutrition and Metabolism) acordó en la necesidad de una "clasificación clínica" de la FI con el objetivo de facilitar la comunicación y la cooperación entre los profesionales de la salud. Teniendo en cuenta que no había datos publicados disponibles para utilizarlos como punto de partida, el desarrollo de una "clasificación clínica" se basó en la experiencia común del panel de expertos, llegando al consenso de clasificar a la FI crónica como enfermedad benigna o cáncer activo, además se basa en los requerimientos calóricos y de volumen intravenosos. Como era de esperarse,

\section{Tabla 1: Clasificaciones en falla intestinal.}

$$
\begin{aligned}
& \text { Clasificación } \\
& \text { fisiopatológica } \\
& \\
& \text { Clasificación } \\
& \text { funcional } \\
& \text { (Tipos) }
\end{aligned}
$$
Clasificación
clínica

Elaboración propia de acuerdo a datos consultados. esta clasificación confiere un amplio rango a la distribución de los pacientes y a la variabilidad de las causas fisiopatológicas así como a los requerimientos energéticos.

En el abordaje inicial de la fase aguda en un paciente con $\mathrm{FI}$ es indispensable el control del cualquier foco séptico, el manejo de la pérdida de volumen, llevar una antibioticoterapia específica, un adecuado manejo de heridas y estomas, el control hidroelectrolítico intensivo, la NPT, múltiples procedimientos quirúrgicos derivativos, desbridativos y de drenaje de colecciones, y en algunos casos el trasplante intestinal. Todo esto requiere de un equipo multidisciplinario que debe estar compuesto por médicos intensivistas, internistas, gastroenterólogos, nutriólogos, psicólogos, psiquiatras, personal de rehabilitación, personal de enfermería especializado, cirujanos, radiólogos, nefrólogos, terapeutas respiratorios, infectólogos, trasplantólogos, personal experto en estomas y manejo de heridas, anestesiólogos y otros, dependiendo de las disfunciones específicas de los órganos. ${ }^{11-14}$

\section{ASOCIACIÓN ENTRE MORTALIDAD Y TFI}

Existen pocos estudios que describen la asociación entre el tipo de falla intestinal, cualquiera que sea la clasificación utilizada, y su mortalidad. Sin embargo, basta con hacer una revisión de la bibliografía en distintas fuentes de textos médicos para encontrar las cifras de mortalidad asociadas con cada patología condicionante específica, existen de igual manera muchos artículos que describen, según la experiencia personal de los autores, la asociación entre distintas patologías y su mortalidad como en la enfermedad de Crohn, siendo de 1.1 a $19.9 \%$, la enfermedad inflamatoria intestinal $0.9 \%$, el megacolon tóxico con resección intestinal y las fístulas intestinales $4 \%$ o la isquemia $<$ mesentérica aguda $58 \%$, entre otras. ${ }^{15-19}$

Los pocos estudios específicos en FI describen una tasa de sobrevida de los pacientes en torno a $88-78 \%$ a los tres y cinco años, respectivamente. En otros centros se ha detectado una mortalidad de $16 \% .9,10$ 
Tabla 2: Defunciones.

\begin{tabular}{lllcc} 
& & n (\%) & Porcentaje válido & Porcentaje acumulado \\
\hline \multirow{2}{*}{ Válido } & Sobrevive & $33(62.3)$ & 62.3 & 62.3 \\
& Finado & $20(37.7)$ & 37.7 & 100.0 \\
& Total & $53(100.0)$ & 100.0 & \\
\hline
\end{tabular}

Elaboración propia de acuerdo a datos consultados.

\section{MATERIAL Y MÉTODOS}

Se realizó un estudio transversal retrospectivo, previa aprobación del Comité de Ética del Hospital Central del Estado; se analizaron las bases de datos de la unidad de falla intestinal (UFI) obteniendo un total de 53 pacientes, los cuales habían ingresado a la unidad durante el periodo comprendido de marzo de 2016 a marzo de 2018, quienes cumplían con los criterios para ser incluidos en el presente estudio (haber sido admitidos en la unidad de falla intestinal y contar con su expediente físico y electrónico completo para las variables de estudio). El vaciamiento de datos se realizó utilizando el programa Microsoft ${ }^{\circledR}$ Excel, y se utilizó el software SPSS ${ }^{\circledR}$ (IBMTM Statistical Package for the Social Science versión 22.0) (SPSS, Chicago, IL, EUA) para el análisis de la información.

\section{RESULTADOS}

De los 53 pacientes recibidos en nuestra unidad de falla intestinal, 33 de ellos se encuentran hasta la fecha con vida que corresponden a $62.3 \%$ del total (Tabla 2).

De la muestra obtenida y de acuerdo con la clasificación funcional, se observó 34\% (18/53) de pacientes con falla intestinal tipo I, $58.5 \%$ (31/53) de pacientes con FI tipo II y $7.5 \%$ (4/53) de pacientes con FI tipo III (Tabla 3). Según la clasificación fisiopatológica (misma que aborda causas quirúrgicas y no quirúrgicas) en nuestra unidad, 52.8\% (28/53) de pacientes correspondieron a la fístula intestinal, $13.2 \%(7 / 53)$ a obstrucción intestinal y solamente 1.9\% (1/53) a síndrome de intestino corto como causas quirúrgicas de falla intestinal. Mencionando a otras entidades ajenas a las antes descritas (y que por lo general son atendidas por el gastroenterólogo), pero de igual modo forman parte de la clasificación fisiopatológica de la falla intestinal, y sólo como mención de ellas, $26.4 \%(14 / 53)$ correspondieron a dismotilidad intestinal y $5.7 \%(3 / 53)$ a síndromes malabsortivos (Tabla 4).

Separando la mortalidad por tipo de falla intestinal según la clasificación funcional, encontramos que en el caso de los pacientes con FI tipo I, fallecieron $55.6 \%(10 / 18)$, de la FI tipo II fallecieron $25.8 \%(8 / 31)$ y en cuanto a la FI tipo III fallecieron 50\% (2/4).

En torno a la clasificación fisiopatológica, falleció el único paciente clasificado como síndrome de intestino corto, de los pacientes con fístula intestinal fallecieron $21.4 \%$ (6/28), de acuerdo a la literatura en otros centros se detectó una mortalidad de $19.1 \%$ en la fístula intestinal; sin embargo, no se especifica que dichos pacientes cumplieran con la definición de falla intestinal. ${ }^{20}$ La mortalidad de los casos de obstrucción intestinal fue de 14.3\% (1/7). De las causas que atiende el colega gastroenterólogo se observó que los pacientes con

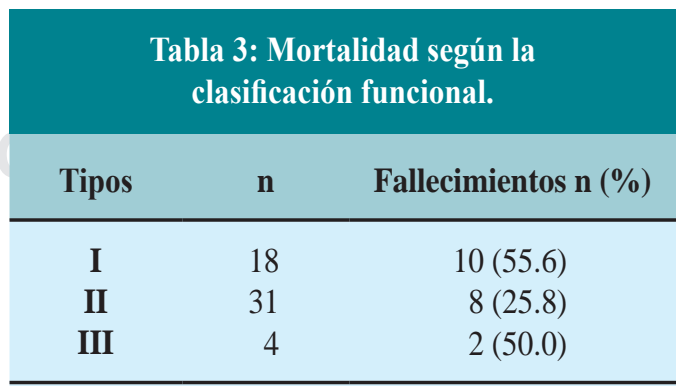

Elaboración propia de acuerdo a datos consultados. 
Tabla 4: Mortalidad según la clasificación fisiopatológica.

\begin{tabular}{lrc} 
& n & Fallecimientos n (\%) \\
\hline Fístula intestinal & 28 & $6(21.4)$ \\
Dismotilidad & 14 & $10(71.4)$ \\
Obstrucción intestinal & 7 & $1(14.3)$ \\
Síndromes malabsortivos & 3 & $2(66.6)$ \\
Síndrome de intestino corto & 1 & $1(100.0)$ \\
\hline
\end{tabular}

Elaboración propia de acuerdo a datos consultados.

dismotilidad intestinal exclusiva, fallecieron $71.4 \%$ (10/14) y por último $66.6 \%$ (2/3) en el caso de enfermedad malabsortiva intestinal. En la revisión de la literatura no se encontraron estudios similares que describieran la tasa de mortalidad de acuerdo con las distintas clasificaciones de falla intestinal.

Entre las principales causas de muerte se observó choque séptico, al que correspondió $50 \%(10 / 20)$ de las defunciones, en su mayor parte siendo secundario a sepsis abdominal difusa causada por perforación intestinal, peritonitis bacteriana, pancreatitis severa o abscesos intraabdominales. La segunda causa con 30\% (6/20) de las defunciones fue la insuficiencia respiratoria aguda principalmente secundaria a cuadro neumónico. Entre otras causas encontramos trombosis mesentérica en 10\% (2/20), fibrilación auricular en 5\% (1/20), y síndrome hepatorrenal secundario a hepatopatía crónica igualmente en 5\% (1/20) (Tabla 5). En estudios similares al presentado se concluye que la causa principal de muerte son las neoplasias de diferente estirpe y en segundo lugar la falla en el manejo hidroelectrolítico. ${ }^{10}$

\section{DISCUSIÓN}

El interés en la insuficiencia intestinal aguda ha aumentado en las últimas décadas y varias publicaciones $^{3-13}$ han informado sobre sus aspectos más relevantes, incluyendo tratamientos y mortalidad. Este estudio retrospectivo de cohorte en pacientes admitidos en nuestro hospital con falla intestinal informa los resultados en cuanto a mortalidad de 53 pacientes, donde se detectó una mortalidad de $37.7 \%$ (20/53) durante el seguimiento por direferentes causas, siendo la principal, sepsis abdominal difusa, manejada con control estricto de líquidos, antibioticoterapia específica por cultivos y aseos abdominales cada 24 a 48 horas; sin embargo, fallecieron, contrastando con la mortalidad de $16 \%$ reportada por Atema y colaboradores. ${ }^{9}$

Todos los pacientes en el presente estudio desarrollaron insuficiencia intestinal en el contexto de una catástrofe intraabdominal, en $52.8 \%$ (28/53) de los casos fue adquirida tras una pérdida de la longitud funcional del intestino asociada con la presencia de una o más fístulas entéricas postoperatorias, cuyo tratamiento ameritó sistemas de vacío artesanales, nutrición parenteral temprana (usualmente con bolsas de la marca Kabiven ${ }^{\circledR}$ o personalizadas del centro de mezclas de la Universidad Autónoma de Chihuahua), lo cual corresponde con los reportes de otros autores que coinciden con esta causa como la principal causante de falla intestinal., ${ }^{7,9}$

De las muertes, $10 \%$ se debieron a trastornos ajenos a la falla intestinal y sus procesos fisiopatológicos incluyendo: fibrilación auricular en 5\% (1/20), y síndrome hepatorrenal secundario a hepatopatía crónica igualmente en $5 \%(1 / 20)$.

\section{CONCLUSIONES}

En nuestro medio la prevalencia de las causas de falla intestinal es similar a la literatura mundial encontrada; sin embargo, la mortalidad y su relación a sepsis abdominal son elevadas, por lo cual descubrimos un área de oportunidad para la reducción de la tasa de mortalidad en nuestros pacientes.

Tabla 5: Causas de mortalidad general.

\begin{tabular}{lr} 
& n \\
\hline Choque séptico & 10 \\
Insuficiencia respiratoria aguda & 6 \\
Trombosis mesentérica & 2 \\
Fibrilación auricular & 1 \\
Síndrome hepatorrenal & 1 \\
\hline
\end{tabular}

Elaboración propia de acuerdo a datos consultados. 


\section{REFERENCIAS}

1. Pironi L, Hébuterne $X$, Van Gossum A, Messing B, Lyszkowska M, Colomb V, et al. Candidates for intestinal transplantation: a multicenter survey in Europe. Am J Gastroenterol. 2006; 101: 16331643.

2. Fleming $C R$, Remington $M$. Intestinal failure. In: Hill GI, editor. Nutrition and the Surgical Patient Clinical Surgery International. Edinburgh, Churchill Livingstone; 1981; 2: 219-235.

3. Rhoda KM, Parekh NR, Lennon E, Shay-Downer C, Quintini C, Steiger E, et al. The multidisciplinary approach to the care of patients with intestinal failure at a tertiary care facility. Nutr Clin Pract. 2010; 25: 183-191.

4. Shaffer J. Intestinal failure: definition and service development. Am J Clin Nutr. 2002; 21: 144e5.

5. Rudolph JA, Squires R. Current concepts in the medical management of pediatric intestinal failure. Curr Opin Organ Transplant. 2010; 15: 324-329.

6. Pironi L, Arends J, Baxter J, Bozzetti F, Peláez RB Cuerda $\mathrm{C}$, et al. ESPEN endorsed recommendations. Definition and classification of intestinal failure in adults. Am J Clin Nutr. 2015; 34: 171-180.

7. Carlson GL, Gardiner K, McKee R, MacFie J, Vaizey C. The surgical management of patients with intestinal failure. Issues in professional practice. Association of Surgeons of Great Britain and Ireland; 2010.

8. Alberti G, Le Roy C, Cofré C, Pattillo JC, Domínguez P, Guerra JF. Actualización en el manejo de pacientes con insuficiencia intestinal Rev Chil Pediatr. 2014; 85: 148-156.

9. Atema JJ, Mirck B, Van Arum I, Ten Dam SM, Serlie MJ, Boermeester MA. Outcome of acute intestinal failure. Br J Surg. 2016; 103: 701-708.

10. Vantini I, Benini L, Bonfante F, Talamini G, Sembenini C, Chiarioni G, et al. Survival rate and prognostic factors in patients with intestinal failure. Dig Liver Dis. 2004; 36: 46-55.

11. Klek S, Forbes A, Gabe S, Holst M, Wanten G, Irtun O, et al. Management of acute intestinal failure: A position paper from the European Society for Clinical Nutrition and Metabolism (ESPEN) Special Interest Group. Am J Clin Nutr. 2016; 35: 1209-1218.

12. Pironi L, Arends J, Bozzetti F, Cuerda C, Gillanders $\mathrm{L}$, Jeppesen PB, et al. ESPEN guidelines on chronic intestinal failure in adults. Clin Nutr. 2016; 35 (2): 247-307. doi: 10.1016/j.clnu.2016.01.020.

13. ESCP Intestinal Failure Group; Vaizey CJ, Maeda Y, Barbosa E, Bozzetti F, Calvo J, et al. European Society of
Coloproctology consensus on the surgical management of intestinal failure in adults. Int J Colorectal Dis. 2016;18: 535-548.

14. Roskott AM, Groen H, Ring EH, Haveman JW, Ploeg RJ, Serlie MJ, et al. Cost-effectiveness of intestinal transplantation for adult patients with intestinal failure: a simulation study. Am J Clin Nutr. 2014; 101: 79-86.

15. Yasukawa S, Matsui T, Yano Y, Sato Y, Takada Y, Kishi $M$, et al. Crohn's disease-specific mortality: a 30-year cohort study at a tertiary referral center in Japan. J Gastroenterol. 2019; 54: 42-52.

16. Sewell JL, Yee HF Jr. 13-year mortality trends among hospitalized patients with inflammatory bowel disease. BMC Gastroenterol. 2012; 12: 79.

17. Doshi R, Desai J, Shah Y, Decter D, Doshi S. Incidence, features, in-hospital outcomes and predictors of inhospital mortality associated with toxic megacolon hospitalizations in the United States. Intern Emerg Med. 2018; 13: 881-887.

18. Tam MS, Abbass M, Tsay AT, Abbas MA. Outcome of colonic fistula surgery in the modern surgical era. Tech Coloproctol. 2014; 18: 467-472.

19. Leone M, Bechis C, Baumstarck K, Ouattara A, Collange $\mathrm{O}$, Augustin $\mathrm{P}$, et al. Outcome of acute mesenteric ischemia in the intensive care unit: a retrospective, multicenter study of 780 cases. Intensive Care Med. 2015; 41: 667-676.

20. Quinn M, Falconer S, McKee RF. Management of Enterocutaneous Fistula: Outcomes in 276 Patients. World J Surg. 2017; 41: 2502-2511.

Consideraciones y responsabilidad ética: Privacidad de los datos. De acuerdo a los protocolos establecidos en el centro de trabajo de los autores, éstos declaran que han seguido los protocolos sobre la privacidad de datos de pacientes preservando su anonimato.

Financiamiento: No se recibió apoyo financiero para la realización de este trabajo.

Conflicto de intereses: Los autores declaran que no existe ningún conflicto de intereses en la realización del trabajo.

\section{Correspondencia:}

Dr. Luis Bernardo Enríquez-Sánchez

E-mail: investigacionhcu@gmail.com 